\title{
Perfusion of Multiple Micronutrients Supplement of HCC-Invaded Human Liver Reduces Oxidative Stress and Proliferation of Cancer Cells
}

\author{
Torricelli Piera ${ }^{1}$, Ferorelli Pasquale ${ }^{2}$, Shevchenko Anna $^{3}$, Antonelli Francesco ${ }^{2}$, Siciliano Alberto $^{2}$, \\ De Martino Angelo ${ }^{2}$, Beninati Simone ${ }^{2}$ \\ ${ }^{1}$ Department SPES, University of Molise, Campobasso, Italy \\ ${ }^{2}$ University of Tor Vergata, Department of Biology, Rome, Italy \\ ${ }^{3}$ People's Friendship University of Russia, Moscow, Russia
}

Email adress:

beninati@bio.uniroma2.it (B. Simone)

\section{To cite this article:}

Torricelli Piera, Ferorelli Pasquale, Shevchenko Anna, Antonelli Francesco, Siciliano Alberto, De Martino Angelo, Beninati Simone. Perfusion of Multiple Micronutrients Supplement of HCC-Invaded Human Liver Reduces Oxidative Stress and Proliferation of Cancer Cells. Cancer Research Journal. Vol. 4, No. 5, 2016, pp. 69-72. doi: 10.11648/j.crj.20160405.11

Received: August 4, 2016; Accepted: August 26, 2016; Published: September 21, 2016

\begin{abstract}
Administration of the chemotherapeutic agent by organ perfusion is more effective than the systemic administration. The active drug is reached by the tumor, to a higher concentration, and normal tissues are spared from major damages. The perfusion of various areas of the body is a recent goal of the experimental and clinical trials. Fatal malignancies observed in humans occur mainly in the abdominal area of the body, The development of a clinically applicable technique for the perfusion of this area is desirable. This report covers the laboratory studies, carried out in the development of a liver perfusion technique for chemotherapy. The results obtained have allowed us to highlight that, the potential anticancer activity of Citozym, a micronutrients supplement, is likely the result of the inhibition of oxidative stress. This work is the preliminary evidence, of the possible mechanism by which Citozym exerts its antineoplastic activity.
\end{abstract}

Keywords: Antioxidants, Organ Perfusion, Oxidative Stress, Micronutrients, HCC, Hepatocarcinoma

\section{Introduction}

Organs or small explants can be removed in vivo and maintained in culture for long periods, if particular attention is paid to the composition of the culture media, the selection of the substrate, and the incubation atmosphere. The explant organ models offer several benefits, relevant to studies of pathophysiological mechanisms, such as cell damage, secretion, differentiation and the development of tumors. In human tissues, obtained from autopsy or surgery, additional attention should be paid to the range post-mortem, the temperature, the moisture and the cause of death. Currently, cultured explant organs, invaded by malignant tumors, are used in our lab, to determine the possibilities of direct action of antineoplastic agents, in an environment, characterized by various types of cells and tissues. In a previous report we proposed, to study the potential antineoplastic in vivo activity, of a food supplement, commercially available as Citozym (CIZ), which have highlighted a cellular protective activity in vitro [1]. This report is concerned with the laboratory studies, carried out in the development of a technique for isolated abdominal organs perfusion. The main aim of this novel approach is to avoid gastric degradation of the active molecules, using as a target directly the organ affected by cancer. Such testing should provide new evidences on the high efficiency of the agent administered directly, into the circulation, which then should be much more active than usual respect oral administration. These tests will be valuable in order to propose future clinical trials. On the basis of earlier experiments, performed on liver explants of C57BL6 / N mice [2], the culture medium used for the perfusion of explants of human liver, invaded by hepatocarcinoma was enriched with a $15 \%$ final concentration of CIZ. It is very important to consider that livers, cold preserved during variable periods of ischemia, suffer functional, morphological and hemodynamic 
alteration, which are exacerbated when they are perfused. One important injury is glycogen depletion during cold ischemia/perfusion. How liver can restore their energy, during perfusion, is related with the preservation time, nutritional status of the donor, and the preservation solution used. However, there are some treatments that help livers to preserve their energy storage. It is feasible that the highly antioxidant solution of CIZ, rich in essential elements for cell metabolism, is able to protect the liver, by reducing the oxidative stress, during visceral perfusion.

Functional status and cell viability can be checked by evaluating the levels of lactate dehydrogenase (LDH) released into the perfusion medium. LDH is involved in tumor initiation and metabolism. Cancer cells rely on increased glycolysis resulting in increased lactate production instead of aerobic respiration in the mitochondria, even under oxygen-sufficient conditions [3]. This state of fermentative glycolysis is catalyzed by the A form of LDH. This mechanism allows tumorous cells to convert the majority of their glucose stores, into lactate, regardless of oxygen availability, shifting use of glucose metabolites from simple energy production to the promotion of accelerated cell growth and replication. It has been reported that upregulation of LDH ensures both an efficient anaerobic glycolytic metabolism and in hypoxic conditions, a reduced dependence on oxygen of the tumor cells The role of LDH in hepatocellular carcinoma (HCC) has been investigated in heterogeneous populations of patients. The levels of plasmatic LDH is considered a potential biomarker in retrospective, small, and nonfocused studies in patients undergoing surgery, transarterial chemoembolization (TACE), and systemic therapy [3].

For this reason, LDH and the possibility of inhibiting its activity, has been identified as a promising target in cancer treatments focused on preventing carcinogenic cells from proliferating. Hence, the preservation solution has an important role in reducing liver damages, during cold ischemia/perfusion and in restoring liver energy storage. In conclusion, the aim of this work is to present studies performed on liver explants and whole organs by different preservation/perfusion media to test the possible improvement of the antineoplastic potential of CIZ on livers, explanted and cultured for several days.

\section{Experimental Protocol}

\subsection{Patients}

Four liver transplanted living-donor patients with a median age at of 59 years (range; 52-68 years), were the sourse of explanted livers affected with HCC lesions. The specimen were considered as surplus of surgical tissue and donate by Prof. Kisinawa (Chiba Surgical Clinic, Chiba, Japan). Two liver explants were tested under perfusion with $15 \% \mathrm{CIZ}$, and two liver explants were perfused with the same serum-free perfusion medium without CIZ, but with $15 \%$ sucrose. The experimental procedures were conducted according to the principles expressed in the Recommendation R (2006) 4 of the European Council, on the Biological Materials of Human Origin (Strasbourg - 15 March 2006).

\subsection{Liver Cultures and Perfusion}

The explanted organs were histologically defined as invaded with hepatocellular carcinoma BCLC staging 5- C [4-5], washed with saline solution (PBS 2\%) and placed in culture in the presence of continuous oxygenation. Liver explants were immersed in $600 \mathrm{~mL}$ of a defined serum-free medium and incubated for 10 days at $37{ }^{\circ} \mathrm{C}$ in a humidified chamber ( $5 \%$ C02). Defined serum-free perfusion medium composition, was DMEM/F12 supplemented with $15 \mathrm{mM}$ HEPES, sodium bicarbonate $45 \mathrm{mM}$ (pH 7.45) and transferrin $6 \mathrm{mM}$, selenium $7 \mathrm{mM}$, insulin $8 \mathrm{mM}$, triiodothyronine $2 \mathrm{mM}$, prostaglandin E1 $6 \mathrm{mM}$ and 100 $\mathrm{IU} / \mathrm{mL}$ of Penicillin and $100 \mathrm{ug} / \mathrm{mL}$ of Streptomycin without fungicide.

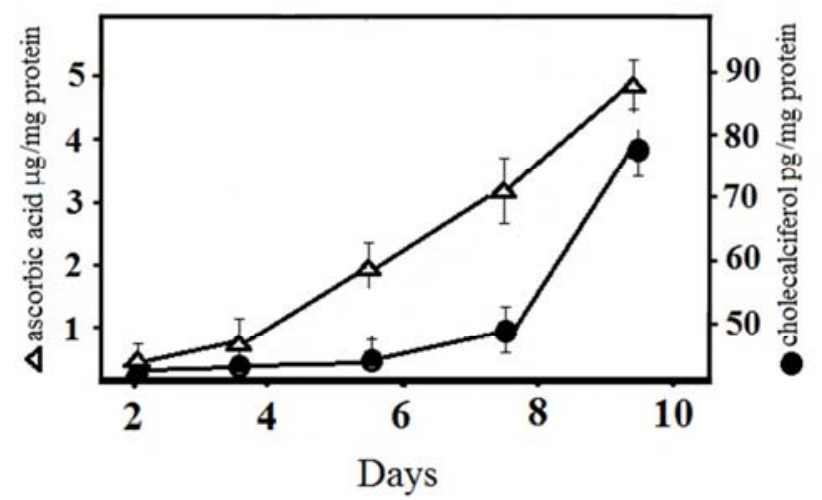

Figure 1. Levels of ascorbic acid and cholecalciferol in perfused liver explants in the presence of CIZ. The value is the average of four determinations \pm S.D.

The liver explants were perfused with the serum-free medium supplemented with $15 \% \mathrm{CIZ}$, while the control group was treated with $15 \%$ sucrose. Complete perfusion medium was changed every 2 days and aliquots were collected throughout the experiment. CIZ was obtained from CITOZEATEC, S.r.l. (Peschiera Borromeo, Milano, Italy). The main components of CIZ are as follows (units/100 g): $500 \mathrm{mg}$ of vitamin $\mathrm{C} ; 56 \mathrm{mg}$ of vitamin B5; $56 \mu \mathrm{g}$ of vitamin D; 3, $3 \mathrm{mg}$ of vitamin B9; $222 \mathrm{mg}$ of pyruvic acid; $120 \mathrm{mg}$ of citric acid; $250 \mathrm{mg}$ of tartaric acid and $77.8 \mathrm{~g}$ of carbohydrates.

\subsection{Bioassays}

The release of lactate dehydrogenase (LDH) from the liver into the medium, was considered as a marker of cell damage. Aliquots of the perfusion medium $(1 \mathrm{~mL})$ were collected every two days and tested for LDH. The test was performed using the Cyto Tox-ONETM homogeneous Membrane Integrity Assay Kit (Promega, Madison, USA). Detection of intracellular ROS levels was performed by using the cellular 2'7-dichlorodihydrofluorescin diacetate (DCFH-DA) assay, based on the description by Rushton and co-workers [6].

The fluorescence of the samples was read at $\lambda 590 \mathrm{ex}$ and 
$\lambda 560 \mathrm{em}$. Evaluation of the levels of two representative CIZ components in cultured liver explant was carried out analyzing the amount of ascorbic acid and cholecalciferol (Figure 1), as markers, in aliquots of the parenchyma of liver explant by HPLC methods [7, 8].

\subsection{Histopathological and Immunohistochemical Analyses}

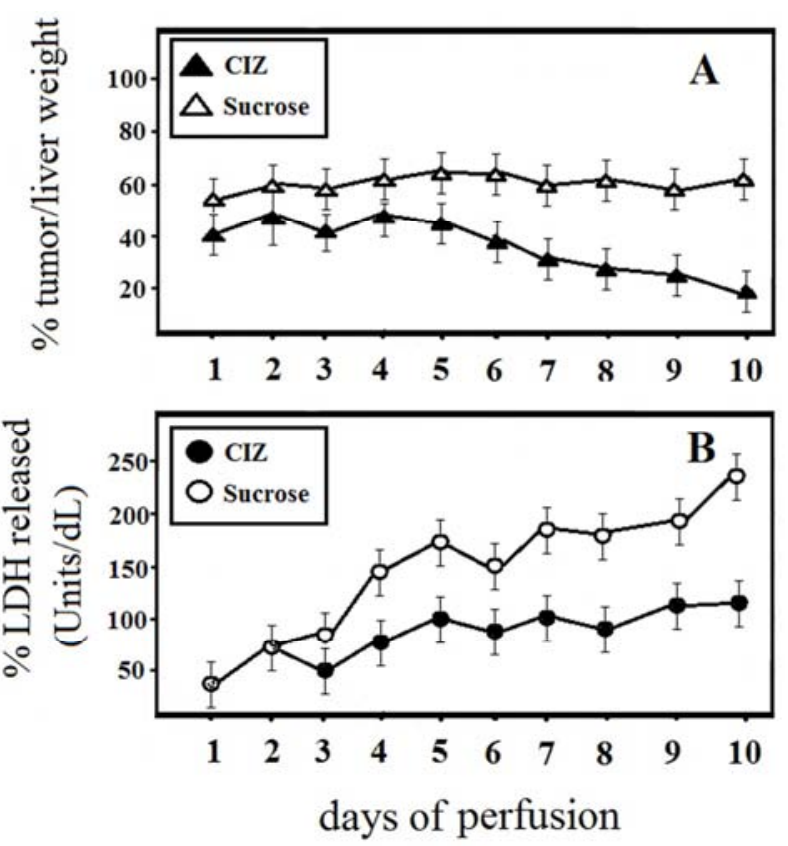

Figure 2. A. Decrease of tumor/liver weight percentages, during the treatment of liver explants, with $15 \%$ CIZ as reported in Material and Methods. B. Levels of $L D H$ released from perfused explants as a marker of cell damage. The values are the average of two determinations \pm S.D.

During the 10 days of perfusion, paraffin-embedded sections of biopsies of explanted livers were prepared by using routine procedures, for histopathological and immunohistochemical examinations. Four $\mu \mathrm{m}$-thick sections of formalin-fixed and paraffin-embedded livers were prepared and stained with hematoxylin and eosin for histopathology. The multiplicity of hepatocellular lesions termed foci of cellular alteration, were assessed on a per unit area $\left(/ \mathrm{cm}^{2}\right)$ basis and determined according to the criteria described by Frith et al. [9].

\section{Results}

It was possible to confirm that perfused liver explants, invaded by $\mathrm{HCC}$, obtained from transplanted donors, are sufficiently viable for more than a week (10 days).

As already reported [2], two main components of CIZ (Vitamin C and D) are absorbed by the explants, in varying quantities. The weight of the liver explants plotted with the weight of the HCC mass, showed a $50 \%$ reduction of the total tumor volume, in samples perfused with $15 \% \mathrm{CIZ}$ for 10 days (Figure 2A). Levels of $\mathrm{LDH}$, released into the perfusion medium was rapidly increased in sucrose treated livers, while were lowered and maintained almost costant, during the 10 days of perfusion with CIZ, indicating the viability of liver explants (Figure 2B). Microscopic examination of the tumor tissues demonstrated that normal hepatic structures had been destroyed by invasion of cancer cells (data not shown).

Reactive oxygen species (ROS) play an important role in cancer growth. Although low levels of ROS can be beneficial, excessive accumulation can promote cancer [10].

Contrary to normal cells, cancer cells produce increased numbers of ROS with a strict dependence on an antioxidant defense system. ROS production plays pivotal roles in mediating endothelial cell apoptosis and regulating the development of atherosclerosis [11], while NADPH oxidase is a major source of vascular ROS production, and the enzyme complex of NADPH oxidases are considered a major source of superoxide anion formation [12]. In addition, NADPH oxidase is also heavily involved in endothelial apoptosis [13]. The presented results indicate that CIZ suppressed ROS production, which helps to elucidate the underlying mechanism for the antineoplastic effect of this multiple micronutrients supplement on HCC-invaded liver explants (Figure 3).

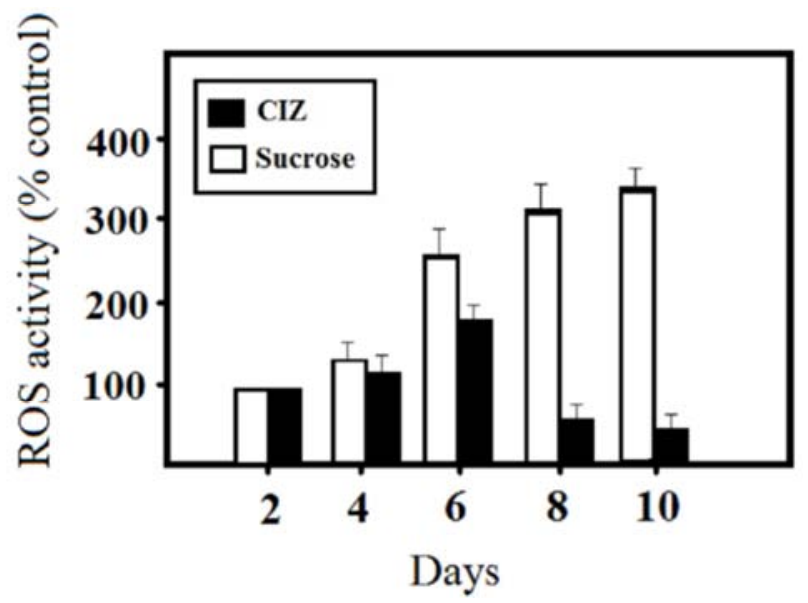

Figure 3. Decrease of ROS activity percentages during the treatment of liver explants with $15 \%$ CIZ compared with the activity of controls as reported in Material and Methods, The values are the average of four determinations $\pm S . D$.

\section{Discussion}

Hepatocellular carcinoma (HCC) is the most common primary liver tumour and represents more than $6 \%$ of all cancers. Although in recent years the therapeutic options for these patients have increased, clinical results are yet unsatisfactory and the prognosis remains dismal.

CIZ, which contains multiple active factors, is a micronutrients dietary supplement, proved useful in reducing the symptoms of various diseases, such as experimental liver steatosis and benign prostatic hyperplasia [14-15].

An antioxidant is a substance that delays, prevents or removes oxidative damage to a target molecule or a compound that directly scavenges ROS or indirectly acts to up-regulate antioxidant defenses or inhibit ROS production. The role of the reduction of oxidative stress, as the mechanism of action of many antitumor drugs, is acquiring an increasing interest 
[16]. In such cases, the antitumor therapy success, may be conditioned by the antioxidants present in the human body, which can be endogenous or exogenous by nutritional supplements. Although, cells possess a large repertoire of enzymes and antioxidants, sometimes these agents are insufficient, to normalize the redox state produced by an intense oxidative stress. In these cases, exogenous antioxidant supplements may be required to restore the cell redox homeostasis.

It has been suggested that antioxidant supplementation may protect the development of certain diseases or that it may reverse the oxidative stress produced during their course. In the area of cancer, antioxidants are increasing in importance. Indeed, it has been reported that antioxidants can prevent cancer due to their effects on oxidative stress [17].

We have recently reported that CIZ, protected C57BL6/N mice from melanoma carcinogenesis, when chronically given before the initiation and promotion phases of melanoma cancer [1]. The various components of the CIZ, may act synergistically, amplifying the antioxidant and antineoplastic activity of molecules such as pyruvic acid, vitamins $\mathrm{C}$ (ascorbic acid), D3 (cholecalciferol), B5 (pantothenic acid) and B9 (folic acid) complex. The presented results, clearly indicate that CIZ suppressed ROS production in HCC-invaded liver explants, which are known to be involved in migration and invasion of cancer cells,. This evidence may provide a novel insight into the mechanisms underlying the antineoplastic activity of this multiple micronutrients supplements.

\section{Acknowledgements}

We are grateful to A.I.A.S. Italy, for financial support for studying the possibility of alternative therapies for cancer and Prof. Kisinawa for the kind gift of liver specimen as surplus of surgical tissues.

\section{References}

[1] Antonelli F. \& Beninati S. Enhanced survival of B16-F10 melanoma tumour-bearing C57BL6/N mice treated with a mixture of antioxidants in: Recent Res Devel in Life Sci. 2011; 5: 51-60 Research Signpost, Trivandrum India

[2] Torricelli P, Antonelli F, Ferorelli P, De Martino A, Shevchenko A, and Beninati S. Organ culture model of liver for the study of cancer treatment for hepatocellular carcinoma. Cancer Res J. 2016; 4 (2): 37-42.

[3] Kolev Y., Uetake H., Takagi Y., Sugihara K. Lactate dehydrogenase-5 (LDH-5) expression in human gastric cancer: association with hypoxia-inducible factor (HIF-1 $\alpha$ ) pathway, angiogenic factors production and poor prognosis. Annals of Surgical Oncology. 2008; 15 (8): 2336-2344.
[4] F. Pons, M. Varela, and J. M. Llovet, Staging systems in hepatocellular carcinoma. HPB (Oxford). 2005; 7 (1): 35-41.

[5] Forner A, Reig ME, de Lope CR, Bruix J. Current strategy for staging and treatment: the BCLC update and future prospects. Semin Liver Dis. 2010; 30 (1): 61-74.

[6] Rushton EK, Jiang J, Leonard SS, Eberly S, Castranova V, Biswas P, et al. Concept of assessing nanoparticle hazards considering nanoparticle dosemetric and chemical/biological response metrics. J Toxicol Environ Health A 2010; 73: 445461.

[7] Moreno P, Salvadó V. Determination of eight water- and fat-soluble vitamins in multi-vitamin pharmaceutical formulations by high-performance liquid chromatography. J Chromatogr A. 2000870 (1-2): 207-215.

[8] Romero Rodriguez MA, Vazquez Oderiz ML, Lopez Hernandez J, Simal Lozano J. Determination of vitamin C and organic acids in various fruits by HPLC. J Chromatogr Sci. 1992; 30 (11): 433-437.

[9] Frith CH, Ward JM, Turusov VS. Tumours of the liver. IARC Sci Publ. 1994; (111): 223-69.

[10] Prasad S, Gupta SC, Tyagi AK. Reactive oxygen species (ROS) and cancer: Role of antioxidative nutraceuticals. Cancer Lett. 2016, doi: 10.1016/j.canlet.2016.03.042. [Epub ahead of print]

[11] Ying Wang, Ira Tabas. Emerging roles of mitochondria ROS in atherosclerotic lesions: causation or association? J Atheroscler Thromb. 2014; 21 (5): 381-390.

[12] O'Brien W J, Farhan Rizvi. NADPH oxidase expression and production of superoxide by human corneal stromal cells $\mathrm{H}$. Mol Vis. 2009; 15: 2535-2543.

[13] Li G, Scull C, Ozcan L, Tabas I NADPH oxidase links endoplasmic reticulum stress, oxidative stress, and PKR activation to induce apoptosis. J Cell Biol. 2010; 191 (6): $1113-1125$.

[14] Torricelli P., Ferorelli P., De Martino A., Antonelli F., Beninati S.. The Influence of Preventive Multiple Micronutrients Supplementation on Liver Steatosis in High- cholesterol Fed C57BL6/N Mice. American Journal of Life Sciences. 2013; 1 (2): 55-60.

[15] Torricelli P., Ferorelli P., De Martino A., Antonelli F. Beninati S.. Preventive Effects of A Mixture of Micronutrients with Antioxidative Properties on Experimentally Induced Prostate Hyperplasia, American Journal of Life Sciences. 2013; 1 (1): 22-26.

[16] Martínez ME, T. Jacobs E, Baron JA, Marshall JR, Byers TJ. Dietary Supplements and Cancer Prevention: Balancing Potential Benefits Against Proven Harms. Natl Cancer Inst. 2012; 104 (10): 732-739.

[17] Thanan R, Oikawa S, HirakuY, Ohnishi S, Ma N, Pinlaor S, Yongvanit P, Kawanishi S, Murata M. Oxidative Stress and Its Significant Roles in Neurodegenerative Diseases and Cancer. Int J Mol Sci. 2015; 16 (1): 193-217. 